Document downloaded from:

http://hdl.handle.net/10251/70517

This paper must be cited as:

Gozálvez Serrano, D.; David Vargas; David Gomez-Barquero; Cardona Marcet, N. (2011). Time Diversity in Mobile DVB-T2 Systems. IEEE Transactions on Broadcasting. 57(3):617628. doi:10.1109/TBC.2011.2161189.

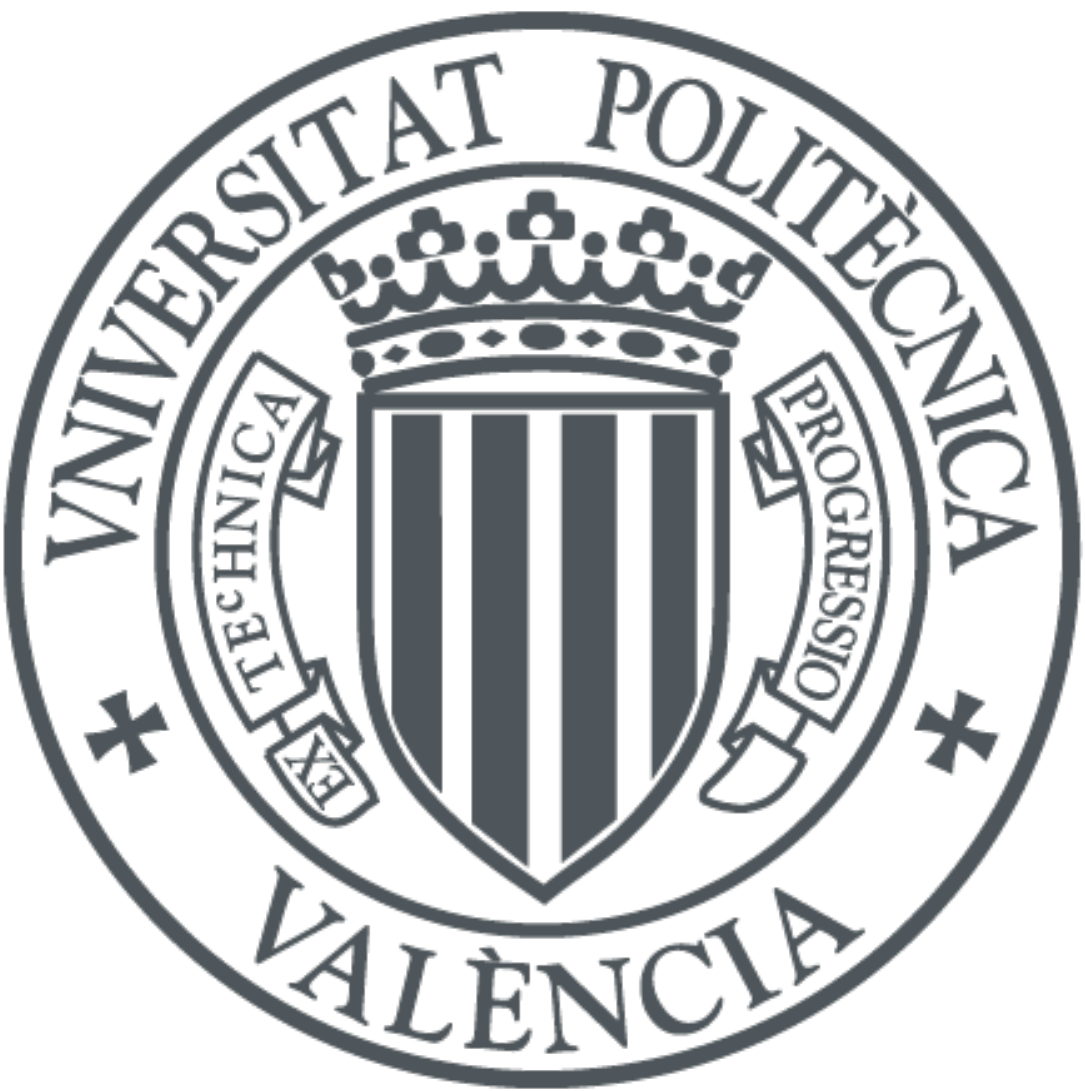

The final publication is available at

http://dx.doi.org/10.1109/TBC.2011.2161189

Copyright Institute of Electrical and Electronics Engineers (IEEE)

Additional Information 


\title{
Time Diversity in Mobile DVB-T2 Systems
}

\author{
David Gozálvez, David Gómez-Barquero, David Vargas and Narcís Cardona
}

\begin{abstract}
DVB-T2 implements a very flexible time interleaving that allows multiple tradeoffs in terms of time diversity, latency and power saving. In this paper, we study in detail these tradeoffs in the context of mobile reception. Together with time diversity, we also investigate the impact of reduced time de-interleaving memory and Alamouti-based MISO in the mobile reception of DVB-T2 services. In addition, we propose the utilization of upper layer FEC protection in order to overcome the limitations of the DVB-T2 physical layer for the provision of long time interleaving, and enable fast zapping. The performance is evaluated by means of simulations in mobile channels that include the presence of fast fading and shadowing in the received signal.
\end{abstract}

Index Terms-DVB-T2, channel coding, mobile TV, time diversity, time interleaving.

\section{INTRODUCTION}

The DVB-T2 (Terrestrial 2nd generation) standard [1] was developed by the DVB (Digital Video Broadcasting) project in order to increase the capacity of terrestrial channels and accommodate high definition TV (HDTV) services. DVB-T2 succeeds in achieving a capacity increase of $50 \%$ over its predecessor DVB-T [2]. The first commercial transmissions of DVB-T2 services began in the UK in December of 2009, and since then, Italy, Sweden and Finland have seen the launch of DVB-T2 services. At the same time, advanced trials are taking place in Austria, Denmark, the Czech Republic and Germany among others. Although DVB-T2 primarily targets static and portable reception, it also incorporates time interleaving in order to benefit from time diversity in mobile scenarios. Time interleaving in DVB-T2 can be configured on a service basis and can provide interleaving durations ranging from miliseconds up to several seconds. It also allows different tradeoffs in terms of time diversity, latency and power saving by means of inter-frame interleaving, sub-slicing and frame hopping. In addition, DVB-T2 incorporates advanced transmission technologies such as low density parity check (LDPC) codes, rotated constellations or multiple-input singleoutput (MISO) [3]. DVB-T2 also introduces the concept of physical layer pipes (PLPs) to enable service specific robustness. By means of multiple PLPs it is possible to accommodate multiple use cases, i.e. static, portable and mobile, in the same frequency channel.

Nevertheless, the simultaneous provision of static and mobile DVB-T2 services in the same frequency channel is limited

David Gozálvez, David Gómez-Barquero, David Vargas and Narcís Cardona are with Instituto de Telecomunicaciones y Aplicaciones Multimedia (iTEAM) of Universidad Politécnica de Valencia, e-mail: \{dagoser, dagobar, davarpa, ncardona\}@iteam.upv.es.

This work was supported in part by the Spanish Ministry of Industry, Tourism, and Commerce under the Celtic project Enabling Next Generation Networks for Broadcast Services ENGINES (TSI-020400-2010-108).

The work of David Gozálvez was supported by the FPU grant AP200803293 of the Spanish Ministry of Education. by the fact that the fast Fourier transform (FFT) size and the pilot pattern have to be defined for the entire DVB-T2 transmission. Static services are generally transmitted with large FFTs and sparse pilot patterns in order to achieve a high spectral efficiency in static channels. However, reception at high velocities requires the utilization of smaller FFTs and more dense pilot patterns to cope with the inter-carrier interference (ICI) that is caused by the Doppler spread.

In order to improve the coexistence of static and mobile services in DVB-T2, the DVB is expected to publish a T2mobile specification based on the DVB-T2 standard. The main objective of the new specification is to allow multiple FFT modes and pilot patterns to be employed in the same frequency channel by means of future extension frames (FEF). FEFs can be multiplexed along with regular T2 frames without impacting the operation of legacy DVB-T2 receivers. This way, T2-mobile services can be transmitted in FEFs with small FFTs and dense pilot patterns while traditional static DVB-T2 services can still benefit from large FFTs and sparse pilot patterns. In addition, the new specification also aims at reducing the complexity of T2-mobile receivers by decreasing the size of the time de-interleaving memory (TDI memory), and by limiting the transmission parameters to a subset of the DVB-T2 standard. Lower code rates originated from the DVB-S2 (Satellite 2nd generation) standard are also included in the new specification as a way to extend the coverage of T2-mobile services.

Although the physical layer of DVB-T2 can provide interleaving durations up to several seconds, the provision of long time interleaving in DVB-T2 is limited by the channel change time and the amount of TDI memory in receivers. The time interleaving included in the physical layer of DVB-T2 does not support fast zapping and hence, the channel change time is proportional to the interleaving duration. Usually, it is considered that channel change times longer than 2 seconds are felt as annoying, whereas less than $500 \mathrm{~ms}$ are seen as instantaneous [4]. On the other hand, the amount of TDI memory in DVB-T2 receivers is defined by the standard to approximately $2^{19}$ cells, which is not sufficient to sustain long interleaving durations (e.g. 10 seconds) for typical mobile TV data rates. This limitation is especially significant in the context of the future T2-mobile specification, which is expected to reduce the amount of TDI memory in receivers down to approximately $2^{18}$ cells.

The utilization of long time interleaving in terrestrial networks can improve the robustness of the data in the presence of shadowing, especially for vehicular reception [5], [6]. Upper layer forward error correction (UL-FEC) can be used in DVB-T2 to overcome the limitations of the time interleaver included in the physical layer, and enable the provision of long time interleaving with fast zapping support [7]. UL-FEC 
mechanisms with long interleaving profiles have been already adopted in mobile satellite DVB technologies. This is the case of MPE-iFEC (Multi Protocol Encapsulation - inter-burst Forward Error Correction) [8], which is included in DVBSH (Satellite to Handheld) for the protection of IP (Internet Protocol) packets at the link layer.

In this paper we investigate the use of time diversity in mobile DVB-T2 systems. A preliminary evaluation of the time interleaving scheme included in DVB-T2 was carried out in [9], where a selected number of configurations were investigated by means of physical layer simulations in fast fading scenarios. In [10], the robustness of the signalling path was studied and compared to the corresponding robustness of the data path. The paper concluded that for certain configurations, the robustness of the signalling information in mobile scenarios may not be sufficiently high when compared to the data. Nevertheless, the study performed in [10] did not investigate the utilization of advanced techniques such as inter-frame interleaving, sub-slicing or MISO, and it did not consider the presence of shadowing in the received signal. Furthermore, the results presented in [10] were obtained assuming Genie aided demapping, which may not represent the true performance of real receivers.

Compared to previous investigations, the work presented in this paper describes in detail all the relevant aspects related to the use of time interleaving in DVB-T2. Regarding the simulation results, we have obtained the performance gain achieved by means of inter-frame interleaving and sub-slicing both separately and in a joint manner. In conjunction with these mechanisms, we have also evaluated the impact of reduced TDI memory, Alamouti-based MISO and UL-FEC protection. These results are not available in the literature and represent a very significant contribution in the context of the future T2mobile specification. The performance evaluation of mobile DVB-T2 systems when the received signal experiments both fast fading and shadowing is another important contribution of the paper, as it constitutes a more challenging scenario than regular fast fading channels for the provision of mobile services in terrestrial networks.

The rest of the paper is organized as follows. In Section II, we review the transmission of information in DVB-T2. Section III is dedicated to the time interleaving implemented in the physical layer for the signalling and for the data path. In this section we also discuss the tradeoffs that are related to the use of time diversity and that should be considered for the delivery of mobile DVB-T2 services. The utilization of ULFEC in DVB-T2 for the provision of long time interleaving is presented in Section IV. In Section V we explain the performance evaluation methodology, while in Section VI we show some representative simulation results that illustrate the impact of time diversity in the performance of mobile DVB-T2 systems. Finally we give some concluding remarks in Section VII.

\section{DVB-T2 OVERVIEW}

\section{A. Data Path}

DVB-T2 incorporates a large number of new features over DVB-T in order to provide better robustness, capacity and flexibility [3]. As with its predecessor, DVB-T2 is based on orthogonal frequency-division multiplexing (OFDM). FFT modes with sizes of $1 \mathrm{~K}, 4 \mathrm{~K}, 16 \mathrm{~K}$ and $32 \mathrm{~K}$ OFDM subcarriers have been added to the original $2 \mathrm{~K}$ and $8 \mathrm{~K}$ modes in order to provide a wider selection of network configurations. The utilization of larger FFTs increases the capacity of the system for the same absolute value of the guard interval, as a higher proportion of the OFDM symbols can be devoted to the transport of data. On the other hand, increasing the FFT size has a negative impact in the Doppler performance as a result of the shorter separation between sub-carriers. Compared to DVB-T, it is also possible to transmit more bits in each sub-carrier by means of 256QAM (Quadrature Amplitude Modulation), which has been added to QPSK (Quaternary Phase Shift Keying), 16QAM and 64QAM. The overhead due to channel sampling is also reduced in DVB-T2 by means of multiple pilot patterns. While DVB-T employs a single pilot pattern, DVB-T2 defines 8 different patterns depending on the selected FFT mode and guard interval. This allows the provision of sufficient channel estimation according to the reception scenario while minimizing the pilot overhead. The overhead due to pilot sub-carriers has been reduced compared to DVB-T from $10.6 \%$ down to $1.35 \%, 2.35 \%$, $4.35 \%$ or $8.35 \%$, depending on the selected pilot pattern. Additional techniques such as extended carrier modes and peak-to-average power ratio (PAPR) reduction techniques can also be used in DVB-T2 to increase the capacity and improve the RF power-amplifier efficiency respectively [2].

DVB-T2 signals are arranged as a sequence of T2 frames, which extend across several OFDM symbols and can be configured with a maximum length of $250 \mathrm{~ms}$. Future extension frames (FEFs) have been also included in the standard in order to allow the introduction of future services in DVB-T2 transmissions (e.g. T2-mobile and DVB-NGH (Next Generation Handheld) services). FEFs can be transmitted between T2 frames in a backwards compatible way, i.e. in such a way that the legacy DVB-T2 receivers are not impacted by the introduction of FEFs. Legacy receivers that are not compatible with the service carried within the FEFs can ignore their reception and wait until the arrival of the next compatible $\mathrm{T} 2$ frame.

Regarding channel coding, DVB-T2 inherits the FEC coding scheme from DVB-S2 based on the concatenation of LDPC and $\mathrm{BCH}$ (Bose Chaudhuri Hocquenghem) codes. There are six code rates $(1 / 2,3 / 5,2 / 3,3 / 4,4 / 5$ and 5/6) and two different FEC word lengths (16200 and 64800 bits) supported in DVB-T2 for the data path. The combined use of LDPC and $\mathrm{BCH}$ codes improves the robustness of the transmitted signal compared to the convolutional and Reed-Solomon codes used in DVB-T. The increased robustness provided by the new channel coding can be traded for greater capacity by means of higher code rates and/or higher order constellations. A bit interleaving and a bit-to-cell demultiplexer are placed after the FEC coding in order to randomize the codewords and assign the less protected bits to the more robust positions in the constellation points of 16QAM, 64QAM and 256QAM constellations. This way it is possible to compensate the unequal bit protection performed by the LDPC code. DVB-T2 also 
includes rotated constellations in order to provide additional robustness [11]. After the symbol mapping, cell, time and frequency interleavers are placed in order to ensure an uncorrelated error distribution within the FEC codewords in time and frequency selective channels. DVB-T2 also incorporates a distributed MISO technique that operates across the antennas of different transmitters in order to improve the reception in single frequency networks (SFN). The distributed MISO scheme included in DVB-T2 employs a modified Alamouti code [12] performed in the frequency direction.

While DVB-T was entirely based on the transmission of MPEG-2 transport streams (TS), DVB-T2 also supports generic streams (GS) as input format. The utilization of generic streams provides a more efficient encapsulation of IP packets and results in less overhead due to packet headers. TS or GS packets are encapsulated inside baseband frames (BB frames) before being modulated and transmitted over the air. Each BB frame constitutes a FEC codeword that is independently encoded by the LDPC and BCH codes. The FEC blocks that result from LDPC and $\mathrm{BCH}$ encoding have a fixed size of 16200 or 64800 bits depending on the selected LDPC word length.

DVB-T2 introduces the utilization of PLPs in order to achieve per-service specific robustness. In this regard, DVBT2 defines two different PLP transmission modes. While input mode A only supports the transmission of a single PLP, input mode B allows multiple PLPs to be transmitted in the same frequency channel. In the first case, the different services are multiplexed into one data stream (e.g. TS or GS) and are transmitted in the same PLP over the air. In the second case, each PLP carries one data stream and can be transmitted with a particular set of transmission parameters, including the constellation, the code rate and the time interleaving configuration. Generally, the different PLPs are multiplexed in time slices within the $\mathrm{T} 2$ frames.

\section{B. Signalling Path}

Layer 1 (L1) signalling in DVB-T2 is transmitted inside preamble symbols known as $\mathrm{P} 1$ and $\mathrm{P} 2$ at the beginning of each T2 frame [10]. The P1 symbol is the first OFDM symbol transmitted in the T2 frames, and is intended for fast identification of available T2 signals. At the same time, it also enables the reception of the $\mathrm{P} 2$ symbols in a very robust way. P2 symbols are transmitted right after the P1 symbol and carry the L1 signalling. The number of $\mathrm{P} 2$ symbols per $\mathrm{T} 2$ frame is given by the FFT mode (e.g. 2 P2 symbols are used in the $8 \mathrm{~K}$ FFT mode).

The L1 signalling transmitted in the P2 symbols can be divided in L1-pre and L1-post signalling. The L1-pre signalling enables the reception of the L1-post signalling and is always transmitted with BPSK (Binary Phase Shift Keying) modulation and code rate 1/5. The L1-post signalling enables the reception of the actual data and is transmitted with modulations BPSK, QPSK, 16QAM or 64QAM and code rate 1/2. L1 signalling is protected by the same BCH and LDPC codes used for the data path. In this case, only the short LDPC code (i.e. 16200 bits) can be selected. Shortening and puncturing is used

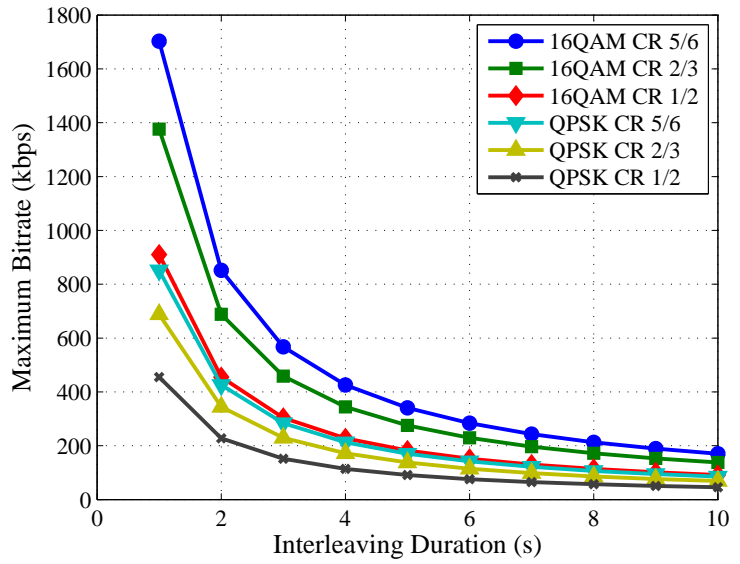

Fig. 1. Maximum PLP data rate supported in DVB-T2 with respect to the interleaving duration for different constellations and code rates.

to adjust the LDPC code to the amount of L1-post information to be transmitted. The codewords containing the L1 signalling information are uniformly distributed over all the P2 symbols of one T2 frame in order to maximize the time diversity. Nevertheless, this accounts for an interleaving duration of just several miliseconds, which may not be sufficient in mobile scenarios.

\section{TIME InTERLEAVING IN DVB-T2}

\section{A. Data Path}

The time interleaver in DVB-T2 consists on a block interleaver that operates on sets of cells referred to as time interleaving blocks (TI blocks). Each TI block corresponds to a different utilization of the TDI memory and is interleaved as a whole by the block interleaver. Since only one TI block is interleaved at a time by the time interleaver, no time interleaving exists between different TI blocks. As a result, the interleaving duration provided by time interleaving in DVB-T2 is equal to the period of time that passes from the transmission of the first and last OFDM symbol carrying cells from the same TI block. Due to the fact that time interleaving in DVB-T2 is performed on a PLP basis, the maximum interleaving duration is limited by the data rate of the PLP and the amount of TDI memory available in receivers. Since the time interleaver is located after the FEC encoder and operates with cells instead of bits, the interleaving duration also depends on the code rate and the constellation. The maximum interleaving duration in DVB-T2 can be computed as:

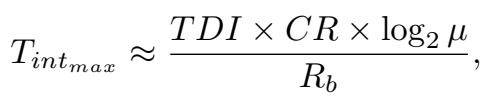

where $T D I$ is the amount of TDI memory, $C R$ is the code rate, $\mu$ is the number of symbols in the constellation (e.g., 4 for QPSK), and $R_{b}$ is the PLP data rate (in bps). The available memory in DVB-T2 receivers for time de-interleaving purposes has been set by the standard to approximately $2^{19}$ cells as a compromise between interleaving capabilities and memory size [1]. In Fig. 1, it is shown the maximum PLP data rate that is supported in DVB-T2 for different combinations of 


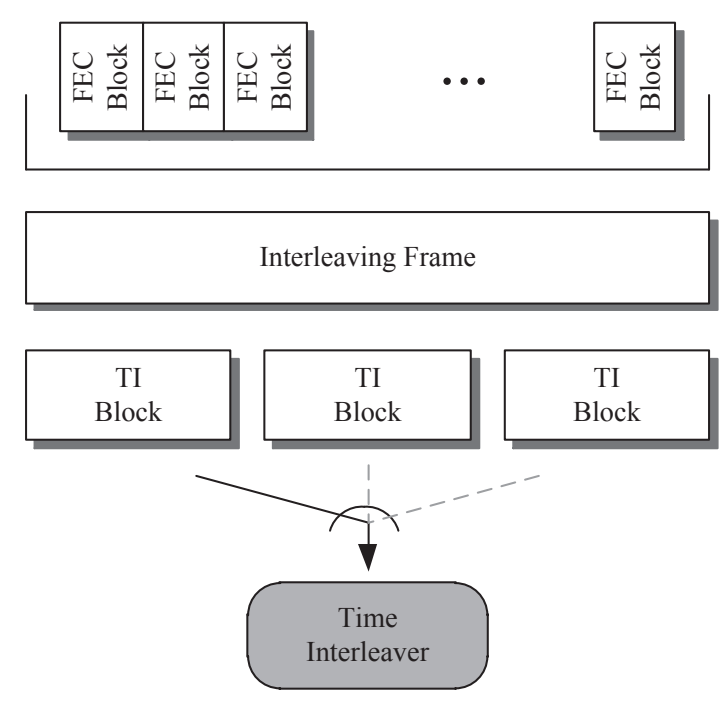

Fig. 2. Time interleaving in DVB-T2. In the figure, one interleaving frame is partitioned into three TI blocks.

constellations and code rates. As can be seen in the figure, long interleaving durations (e.g. 10 seconds) cannot be achieved for typical mobile TV data rates. It should be noted that in the case of T2-mobile, the TDI memory is limited to approximately $2^{18}$ cells and hence, the maximum supported PLP data rate shown in Fig. 1 would be halved for any given interleaving duration.

The PLP data rate is determined in a major way by the PLP input mode. The utilization of input mode B divides the overall data rate among different PLPs. This way, each PLP ends up with a lower individual data rate and can be transmitted with a longer interleaving duration. On the contrary, input mode A collects the entire data rate in a single PLP, which decreases the maximum interleaving duration that can be applied.

The FEC blocks pertaining to the same PLP are grouped in interleaving frames before being passed to the time interleaver. Each interleaving frame consists of a dynamically varying number of FEC blocks. Once the interleaving frames have been processed by the time interleaver, they are mapped to T2 frames for transmission. Depending on the PLP data rate, the amount of data in one $\mathrm{T} 2$ frame may exceed the available TDI memory. In this case, the interleaving frame cannot be deinterleaved as a whole, and it must be partitioned in multiple TI blocks so that each TI block can be processed separately by the time de-interleaver. This allows the transmission of PLPs with a higher data rate, but decreases the maximum interleaving duration as a result. The partition of the interleaving frames in multiple TI blocks is common in the case of input mode A, due to the transmission of a single higher data rate PLP. The arrangement of FEC blocks for time interleaving is illustrated in Fig. 2. In this case, one interleaving frame is partitioned into three different TI blocks that are interleaved by the time interleaver one after the other.

DVB-T2 allows inter-frame interleaving, frame hopping and sub-slicing when mapping the interleaving frames to the $\mathrm{T} 2$ frames. Inter-frame interleaving is achieved when one

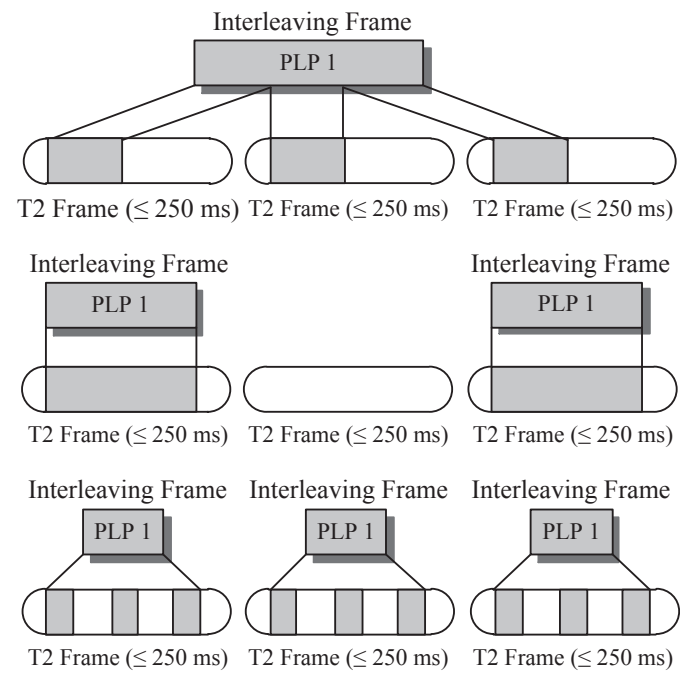

Fig. 3. Frame mapping options in DVB-T2: inter-frame interleaving (top), frame hopping (center) and sub-slicing (bottom).

interleaving frame is transmitted across multiple T2 frames. This is only allowed when the interleaving frame has been interleaved as a single TI block. By means of inter-frame interleaving it is possible to extend the interleaving duration beyond one $\mathrm{T} 2$ frame. It must be noted that the maximum interleaving duration is always limited by (1). An example of inter-frame interleaving is illustrated in Fig. 3 (top), where one interleaving frame is mapped to be transmitted in three $\mathrm{T} 2$ frames.

PLPs configured to perform frame hopping are not transmitted in every $\mathrm{T} 2$ frame but only in a subset of frames regularly distributed over time. The separation between frames carrying information from one PLP is referred to as the frame interval. A frame interval of two T2 frames is represented in Fig. 3 (centre).

DVB-T2 distinguishes between two different types of PLPs according to the number of time slices per frame. While PLPs type 1 are always carried in a single time slice per frame, the PLPs type 2 can be carried in multiples sub-slices. The latter case is known as sub-slicing. Inside the T2 frames, the PLPs type 1 are transmitted prior to the transmission of the PLPs type 2. Although the number of sub-slices per T2 frame is configurable, it is the same for every PLP type 2 carried in the same set of frames. If the number of sub-slices is high enough, the information from each PLP type 2 is transmitted continuously over time within the frames. Only certain values of sub-slicing and combinations with inter-frame interleaving are allowed in the standard. This is due to signalling issues and undesirable interactions with the frequency interleaver that may result in the loss of frequency diversity [2]. It should be noted that the maximum number of allowed sub-slices decreases with higher values of inter-frame interleaving. The transmission of three sub-slices per T2 frame is illustrated in Fig. 3 (bottom). 


\section{B. Signalling Path}

The L1 signalling transmitted in the P2 symbols does not feature time interleaving, and the interleaving duration is restricted to several miliseconds. DVB-T2 includes two mechanisms for increasing the robustness of the L1 signalling known as L1 repetition and in-band signalling. The former increases the robustness of the L1 signalling by transmitting in each $\mathrm{T} 2$ frame the signalling information that corresponds to the current and the next $\mathrm{T} 2$ frame. The latter transmits the L1 signalling embedded in the data path so that it possesses the same robustness as the data. In particular, when in-band signalling is used, the first $\mathrm{BB}$ frame of each interleaving frame carries the L1 signalling corresponding to the next interleaving frame. This way it is possible to improve the continuous reception of the service without the need of receiving the P2 symbols. Although the utilization of L1 repetition and in-band signalling introduces a delay in transmission of one $\mathrm{T} 2$ frame and one interleaving frame respectively, it does not result in an increase of the channel change time.

\section{Time Diversity - Latency Tradeoff}

The time interleaver included in DVB-T2 does not support fast zapping, and short channel change times cannot be achieved with long time interleaving. When switching to a new PLP, DVB-T2 receivers have to wait until the complete reception of one entire TI block before they can de-interleave and process the FEC blocks. Consequently, the channel change time is proportional to the interleaving duration. The longer the interleaving duration, the longer the receivers must wait prior to the de-interleaving of the TI blocks.

In the case of PLPs type 1, all the cells are transmitted contiguously in one single time-slice within the T2 frames. As a result, the intra-frame interleaving duration is limited by the maximum number of cells that fit in the TDI memory. In the case of $2^{19}$ cells, this corresponds to approximately 100 ms. On the contrary, PLPs type 2 employ sub-slicing to spread the cells across the T2 frame and in this case, the interleaving duration is limited by the T2 frame length (up to $250 \mathrm{~ms}$ ).

Inter-frame interleaving can be used with PLPs type 1 and PLPs type 2 to extend the interleaving depth beyond the duration of one $\mathrm{T} 2$ frame. By means of inter-frame interleaving it is possible to achieve interleaving durations up to several seconds at the expense of increased channel change time.

Frame hopping on the other hand increases the channel change time as it delays the transmission of information between non-consecutive $\mathrm{T} 2$ frames. In addition, it may result in an improvement of the intra-frame interleaving. When frame hopping is used, the information from the PLPs must be buffered during one frame interval before it can transmitted over the air. This increases the amount of information to be transmitted in one T2 frame, which results in a higher TDI memory utilization and longer interleaving durations when sub-slicing is not employed.

Assuming that sub-slicing is used, the average channel change time that a receiver must wait until the complete reception of the first interleaving frame can be computed as:

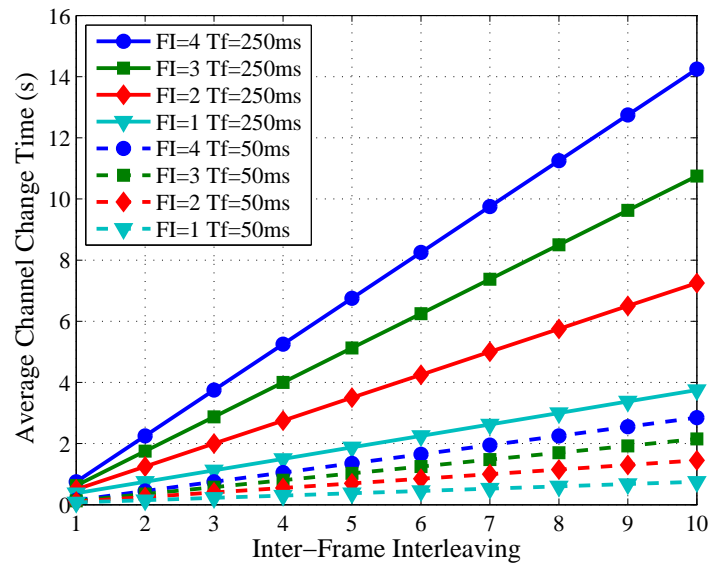

Fig. 4. Average channel change time of DVB-T2 services for different frame intervals and inter-frame interleavings. T2 frame length of $250 \mathrm{~ms}$ and $50 \mathrm{~ms}$.

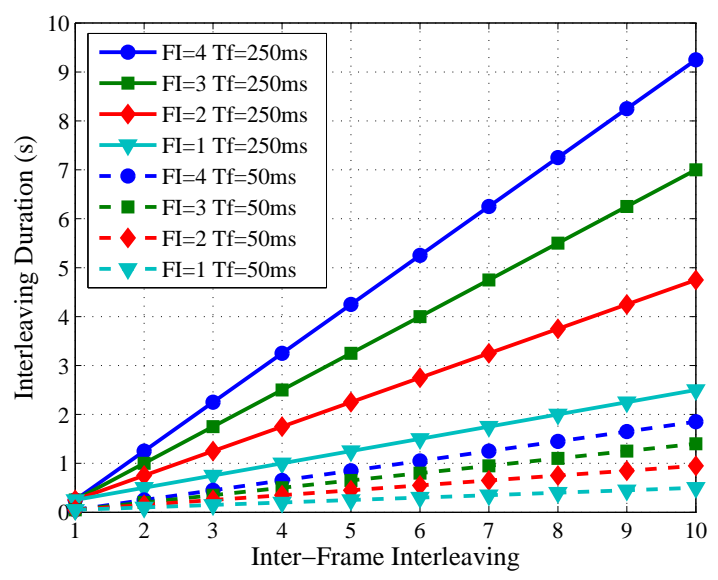

Fig. 5. Interleaving duration for different frame intervals and inter-frame interleavings. T2 frame length of $250 \mathrm{~ms}$ and $50 \mathrm{~ms}$.

$$
T_{\text {change }} \approx T_{\text {wait }}+T_{\text {int }},
$$

where $T_{\text {int }}$ is the interleaving duration and $T_{\text {wait }}$ is the average period of time until the arrival of the first $\mathrm{T} 2$ frame carrying the beginning of an interleaving frame. The value of $T_{\text {wait }}$ is given by:

$$
T_{\text {wait }} \approx \frac{T_{\text {frame }} \times F I \times I F}{2},
$$

where $T_{\text {frame }}$ is the duration of the $\mathrm{T} 2$ frame, $F I$ is the frame interval and $I F$ is the inter-frame interleaving. On the other hand, $T_{i n t}$ can be computed as:

$$
T_{\text {int }} \approx T_{\text {frame }} \times(F I \times(I F-1)+1) .
$$

In Figures 4 and 5 we represent the average channel change time and the interleaving duration respectively with different combinations of frame hopping and inter-frame interleaving, and for T2 frames of $250 \mathrm{~ms}$ and $50 \mathrm{~ms}$. It must be noted that channel change times above 2 seconds are perceived as annoying [4]. As can be seen in the figure, the utilization 
of inter-frame interleaving and frame hopping increases both the interleaving duration and the channel change time in a significant manner. Although higher values of inter-frame interleaving and frame hopping are required to achieve any given interleaving duration in the case of short $\mathrm{T} 2$ frames, the resulting average channel change time is approximately the same as with long frames.

\section{Time Diversity - Power Saving Tradeoff}

DVB-T2 receivers can switch-off their RF components during the periods of time between time slices of the same PLP in order to reduce the power consumption. From a power saving point of view, each PLP should be transmitted in one single time slice of information inside the $\mathrm{T} 2$ frames. The main reason for this is that due to synchronization issues, receivers need to wake up a certain period of time prior to the actual reception of each time slice. On the other hand, the transmission of multiple sub-slices per T2 frame results in a more even distribution of information and achieves a higher time diversity. In fact, the maximum time diversity is achieved when the services are transmitted continuosly over time.

If the number of sub-slices is high enough, receivers cannot perform power saving, and they must have their RF components switched on during the entire $\mathrm{T} 2$ frame. If frame hopping is enabled, receivers can still achieve power saving by skipping the reception of entire $\mathrm{T} 2$ frames and switching off their frontends until the arrival of the next frame with PLP information. This way it is possible to perform power saving even when the PLPs are transmitted continuously within the T2 frames.

The duration of the time slices inside a $\mathrm{T} 2$ frame is given by:

$$
T_{\text {slice }} \approx\left\lceil\frac{N_{\text {cells }}}{N_{\text {data }} \times N_{\text {slices }}}\right\rceil \times T_{\text {symbol }}
$$

where $N_{\text {cells }}$ is the number of cells from the same PLP to be transmitted in the T2 frame, $N_{\text {data }}$ is the amount of data carriers per OFDM symbol, $N_{\text {slices }}$ is the number of subslices per $\mathrm{T} 2$ frame and $T_{\text {symbol }}$ is the duration of the OFDM symbols. In turn, the power consumption in DVB-T2 can be computed as:

$$
P \approx \frac{\min \left\{T_{\text {frame }}, N_{\text {slices }} \times\left(T_{\text {synchro }}+T_{\text {slice }}\right)\right\}}{F I \times T_{\text {frame }}}
$$

where $N_{\text {slices }}$ is the number of slices in one T2 frame, $T_{\text {synchro }}$ is the time required for synchronization, $T_{\text {slice }}$ is the duration of one slice, $F I$ is the frame interval and $T_{\text {frame }}$ is the duration of the $\mathrm{T} 2$ frame.

In Fig. 6 we illustrate the impact of sub-slicing and frame hopping in the power saving capabilities of DVB-T2 receivers. We have represented the case with $\mathrm{T} 2$ frame lengths of 250 $\mathrm{ms}$ and $50 \mathrm{~ms}$. It must be noted that some of the values of subslicing represented in the figure are not allowed in the standard for certain configurations. We have assumed the maximum utilization of the TDI memory (up to $2^{19}$ cells), 6208 data subcarriers per OFDM symbol and an OFDM symbol duration of

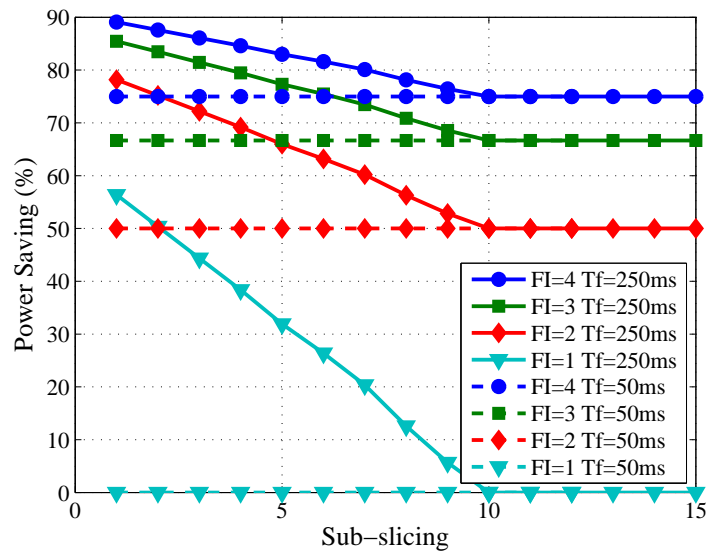

Fig. 6. Power consumption of DVB-T2 services for different frame intervals and sub-slicing values. T2 frame length of $250 \mathrm{~ms}$ and $50 \mathrm{~ms}$.

$1.12 \mathrm{~ms}^{1}$. We have also considered a synchronization time of $15 \mathrm{~ms}$, which is presented in [13] as a typical value of fast synchronization schemes.

In the case of long $\mathrm{T} 2$ frames, the power saving decreases with the number of sub-slices until a certain saturation value. At this point receivers must be powered on during the entire $\mathrm{T} 2$ frame due to the additional synchronization time and thus, power saving does not decrease further with the number of sub-slices. On the contrary, if short T2 frames are used, each frame may be filled with information from one PLP without the need of sub-slicing. In this case, frame hopping is required not only to obtain power saving, but also to multiplex multiple PLPs in the same frequency channel. Each PLP is therefore transmitted in a subset of $\mathrm{T} 2$ frames regularly placed over time, while the rest of frames carry information from different PLPs. This ressembles the transmission of services in DVB-H.

\section{UPPER LAYER FEC PROTECTION IN DVB-T2}

Due to the limited amount of TDI memory available in DVB-T2 receivers (approx. $2^{19}$ cells), long interleaving durations (i.e. several seconds) cannot be provided for typical mobile TV data rates. In addition, the time interleaver included in the physical layer of DVB-T2 does not support fast zapping, and long time interleaving cannot be used with tolerable channel change times. In order to overcome these limitations, UL-FEC protection can be used in DVB-T2 to provide long time interleaving at upper layers with fast zapping support. If fast zapping is enabled, receivers in good reception conditions can start to display the service before the reception of all the information and parity data. Depending on the UL-FEC implementation, it may be still possible to achieve full protection (e.g. the entire information and parity data is available for decoding) later on by means of parity recovery techniques [14].

UL-FEC protection has been standardized in the DVB family of standards in order to improve the robustness of the

\footnotetext{
${ }^{1}$ The number of data sub-carriers corresponds to pilot pattern PP1 and FFT mode $8 \mathrm{~K}$. Likewise, the duration of the OFDM symbols corresponds to FFT mode $8 \mathrm{~K}$ and guard interval $1 / 4$.
} 
transmitted information by extending the interleaving duration of the physical layer. This is the case of DVB-SH, which incorporates MPE-iFEC for the protection of IP datagrams at the link layer [8]. Nevertheless, it is also possible to implement UL-FEC at the application layer of DVB systems in order to provide a similar protection as MPE-iFEC.

A major motivation for UL-FEC is that it is generally possible to implement long time interleaving at upper layers with more relaxed hardware requirements ${ }^{2}$ [7]. Depending on the encoding algorithm employed, it may be also possible to use the general purpose memory for the operation of ULFEC. This avoids the implementation of additional expensive on-chip memory and facilitates the utilization of UL-FEC in DVB-T2 receivers.

In order to optimize the overall system performance when UL-FEC is employed in DVB-T2 systems, the PLPs should be transmitted continuously inside the $\mathrm{T} 2$ frames by means of sub-slicing whereas inter-frame interleaving is performed at upper layers. The main idea is to combine the powerful protection at the physical layer against noise and fast fading, with the long interleaving protection at upper layers against shadowing. If necessary, power saving can be achieved by means of frame hopping. The combination of short time interleaving at the physical layer with long time interleaving at the upper layer has been already included in DVB-SH for its utilization in class 1 receivers [15].

\section{Performance Evaluation Methodology}

We have investigated the use of time diversity in DVB-T2 by means of computer simulations. Table I shows the simulation parameters. We have selected the BB FER (Baseband Frame Error Rate) $1 \%$ as quality of service (QoS) criterion. Bit error ratios (BER) were used to evaluate the system performance in the standardization process of DVB-T2. More specifically, the QoS criterion followed was a BER of $10^{-7}$ after LDPC decoding [2]. However, BER criterions only indicate the percentage of erroneous bits and are not a proper indicator of the QoS seen by upper layers.

The simulations employ the 6-taps typical urban (TU6) channel model, which is representative of mobile reception in fast fading scenarios for Doppler frequencies above $10 \mathrm{~Hz}$. The TU6 channel model has been widely used in the performance evaluation of mobile TV systems like DVB-H [16]. For the MISO simulations we have considered the SFN TU6 channel model. This model has been proposed in the standardization process of DVB-NGH for the evaluation of mobile reception in SFNs [17]. Each transmitter in the SFN is modelled as one independent TU6 profile so that the total mobile SFN channel is the sum of all individual and independent TU6 channel models, each having a unique power level and delay.

\footnotetext{
${ }^{2}$ Contrary to the channel decoding performed at the physical layer of DVB-T2, UL-FEC operates on a packet erasure level. This means that each transmitted packet is considered in reception as either entirely correct or erased. The storage of erasure error information requires considerable less memory than the soft information used in the physical layer of DVB-T2. In particular, each cell requires in the order of 10 bits for each real and imaginary part in addition to some bits for channel state information. On the contrary, the erasure information only needs an additional storage of 1 bit per byte in order to account for erasures on a byte level.
}

TABLE I

SimUlation PARAMETERS

\begin{tabular}{c||c}
\hline \multicolumn{1}{c||}{ System Parameters } & Value \\
\hline \hline Bandwidth & $8 \mathrm{MHz}$ \\
\hline FFT mode & $8 \mathrm{~K}$ \\
\hline Guard Interval & $1 / 4$ \\
\hline Input mode & $\mathrm{B}$ \\
\hline FEC word length & 16200 \\
\hline Code Rate & $1 / 2$ \\
\hline Channel estimation & Perfect \\
\hline QAM demapping & Optimum soft maximum a posteriori (MAP) \\
\hline Channel model & TU6 \\
& SFN TU6 \\
\hline QoS criterion & TU6+shadowing \\
\hline \hline
\end{tabular}

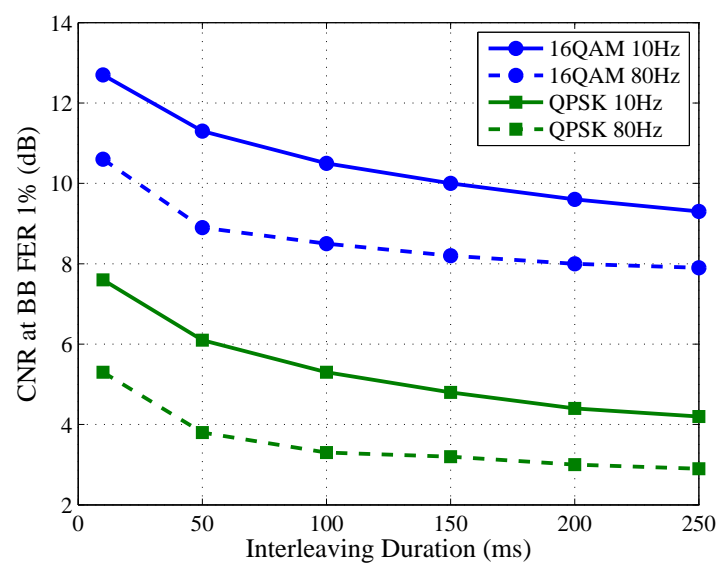

Fig. 7. CNR at BB FER $1 \%$ with intra-frame interleaving in the TU6 channel.

In order to investigate the influence of shadowing, we have assumed a user moving at constant velocity across a lognormal carrier-to-noise (CNR) map defined by its standard deviation $(\sigma)$ and correlation distance $\left(d_{c o r r}\right)$. The user velocity is given by the Doppler frequency $\left(f_{d}\right)$ and the carrier frequency $\left(f_{r f}\right)$. The shadowing model outputs CNR values that are used in the TU6 simulations as the average CNR over time. This way it is possible to combine the presence of fast fading and shadowing in the received signal. In the simulations we have used Doppler frequencies of $10 \mathrm{~Hz}$ and $80 \mathrm{~Hz}$ with a carrier frequency of $600 \mathrm{MHz}$, which correspond to user velocities of $18 \mathrm{~km} / \mathrm{h}$ and $144 \mathrm{~km} / \mathrm{h}$ respectively.

\section{Simulation Results}

\section{A. Fast Fading - Data Path}

We first evaluate the mobile performance of DVB-T2 with intra-frame interleaving, i.e. when the interleaving duration does not exceed the T2 frame length (up to $250 \mathrm{~ms}$ ). Fig. 7 shows the required CNR at BB FER $1 \%$ for interleaving durations ranging from $10 \mathrm{~ms}$ to $250 \mathrm{~ms}$. It must be noted that in the case of intra-frame interleaving with $2^{19}$ cells of TDI memory, it is not possible to achieve interleaving durations higher than $100 \mathrm{~ms}$ without the use of sub-slicing $(50 \mathrm{~ms}$ in 

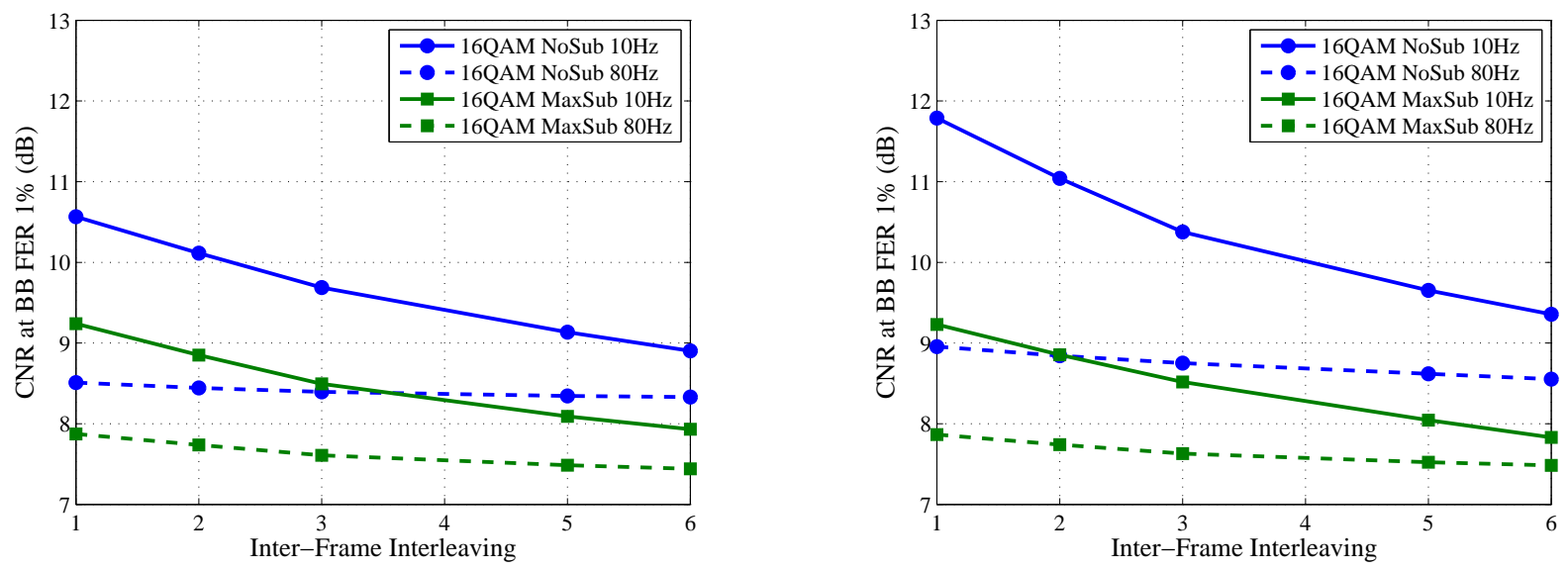

Fig. 8. CNR at BB FER $1 \%$ for different configurations of inter-frame interleaving with and without sub-slicing in the TU6 channel. TDI memory of $2^{19}$ cells (left) and $2^{18}$ cells (right).
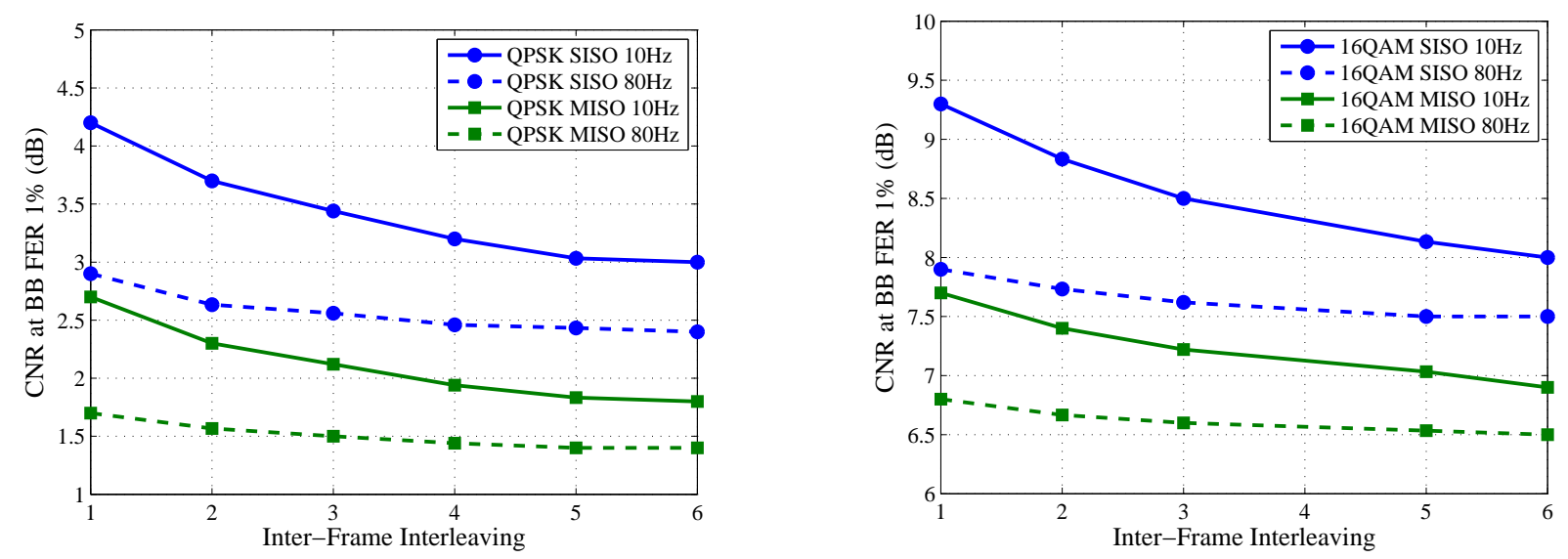

Fig. 9. CNR at BB FER 1\% for different configurations of inter-frame interleaving with and without MISO. SFN TU6 channel with no delay or power imbalance between antennas.

the case of T2-mobile). This value is only obtained when the entire TDI memory is filled with information and generally, the interleaving duration without sub-slicing is considerably lower. On the contrary, if sub-slicing is enabled, interleaving durations higher than $100 \mathrm{~ms}$ can be easily obtained by setting the T2 frame length accordingly. As can be seen in Fig. 7, the difference between an intra-frame interleaving duration of 10 ms and $250 \mathrm{~ms}$ is approximately $3.5 \mathrm{~dB}$ in the case of $10 \mathrm{~Hz}$ of Doppler and $2.5 \mathrm{~dB}$ in the case of $80 \mathrm{~Hz}$. These results represent a very significant gain, and justify the use of subslicing in mobile escenarios.

The performance of sub-slicing in conjunction with interframe interleaving is illustrated in Fig. 8, where we show the results for inter-frame interleaving values ranging from one to six $\mathrm{T} 2$ frames. This corresponds to interleaving durations from $250 \mathrm{~ms}$ to $1.5 \mathrm{~s}$ in steps of $250 \mathrm{~ms}$. It should be noted that the results lack the case with four T2 frames, as it is not allowed in the standard for the short LDPC code and 16QAM. Regarding sub-slicing, we have employed the maximum value allowed by the standard for each configuration.

In the case of $2^{19}$ cells of TDI memory, the gain achieved by sub-slicing ranges from $1.5 \mathrm{~dB}$ down to $1 \mathrm{~dB}$, and generally decreases with higher values of Doppler and inter-frame interleaving. In the case of $2^{18}$ cells, the gain ranges from $2.5 \mathrm{db}$ down to $1 \mathrm{~dB}$. The results show that the reduced TDI memory does not impact the system performance as long as sub-slicing is used. Otherwise, a degradation up to $1 \mathrm{~dB}$ can be expected in low Doppler scenarios.

On the other hand, the gain achieved by an inter-frame interleaving up to six frames (i.e. $1.5 \mathrm{~s}$ ) varies between 2.5 $\mathrm{dB}$ and less than $0.5 \mathrm{~dB}$. As can be seen, the performance of inter-frame interleaving is heavily conditioned by the use of sub-slicing, the TDI memory and the Doppler. In particular, the gain due to inter-frame interleaving is only significant in low Doppler scenarios, especially if sub-slicing is not used and with reduced TDI memory.

In Fig. 9 we show the mobile performance of DVB-T2 when MISO is used in conjuntion with inter-frame interleaving and sub-slicing. We have considered no power imbalance or delay between transmitters in the SFN TU6 channel. This represents the case of maximum spatial diversity in an SFN network. It should be noted that the diversity obtained by 

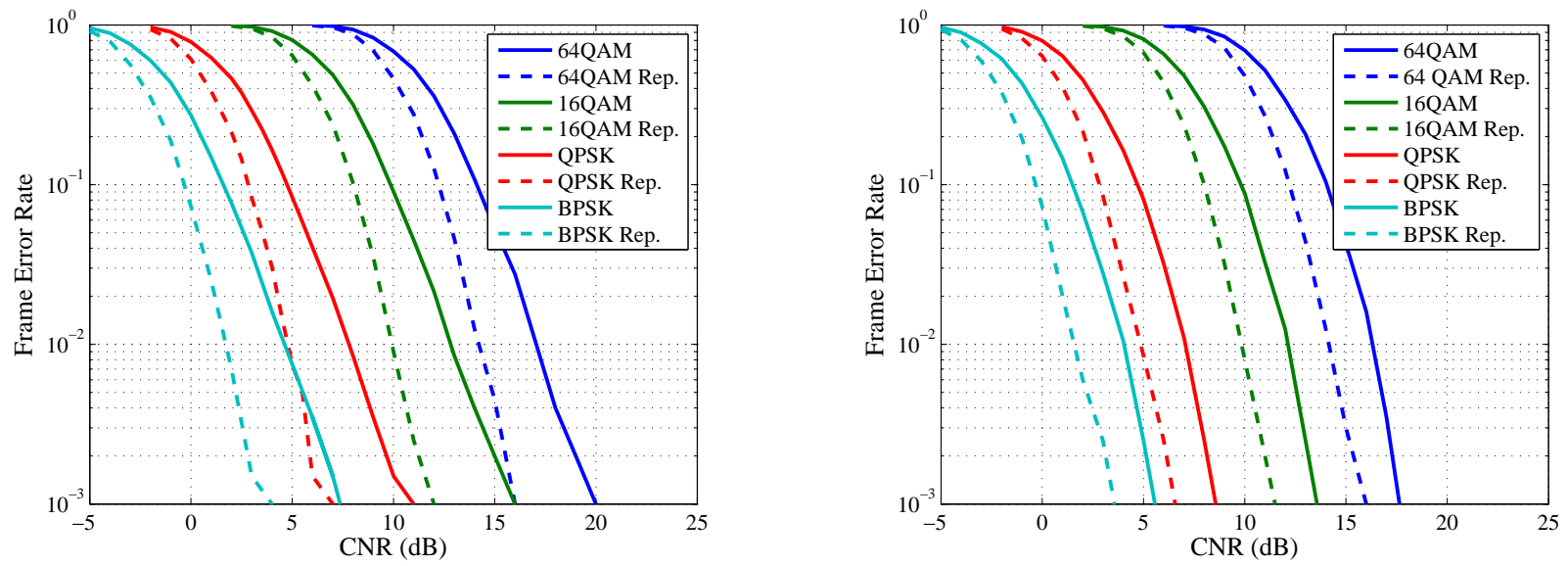

Fig. 10. Frame error rate curves of the L1-post signalling in the TU6 channel. Doppler frequency of $10 \mathrm{~Hz}$ (left) and $80 \mathrm{~Hz}$ (right).

means of Alamouti-based MISO is equal to applying maximalratio receiver combining (MRRC) with two antennas at the receiver [12]. Therefore, the results of Fig. 9 also correspond to the performance obtained with one transmitting and two spatially separated receiving antennas (single-input multipleoutput, SIMO), except for an offset of $3 \mathrm{~dB}$ due to the power $\operatorname{loss}^{3}$. The utilization of SIMO is very interesting in the context of vehicular reception, where multiple antennas with sufficient spatial separation can be easily implemented at the receiver side.

From the figures we can see that the utilization of MISO provides a gain between $1 \mathrm{~dB}$ and $1.5 \mathrm{~dB}$ depending on the Doppler frequency and the interleaving duration. In particular, this gain decreases for longer interleaving durations and higher Doppler values. In the fast fading scenario, both time and space diversity benefit from the selectivity caused by multi-path propagation. Due to the diminishing returns of diversity, the great availability of diversity in the time domain may decrease the impact of additional diversity in the spatial domain, and vice versa.

\section{B. Fast Fading - Signalling Path}

The frame error rate curves of the L1-post signalling with and without L1 repetition are plotted in Fig. 10. These results are very similar to those presented in [10]. Small differences are caused by the fact that we have assumed full ocupation of the LDPC codewords and hence, no puncturing or padding has been performed in the encoding process.

From the figures we can see that the gain due to L1 repetition is equal to $2 \mathrm{~dB}$ in the case of $80 \mathrm{~Hz}$ of Doppler and equal to $3 \mathrm{~dB}$ in the case of $10 \mathrm{~Hz}$. If we compare the performance of Fig. 10 with the results of Fig. 9, we can see that the use of $\mathrm{L} 1$ repetition in the signalling path ensures a higher robustness than the data path as long as the signalling is transmitted with BPSK constellation. For example, in Fig. 9

\footnotetext{
${ }^{3}$ The utilization of two receiving antennas results in an array gain of $3 \mathrm{~dB}$ due to the coherent combination of signals. Array gains cannot be obtained with multiple transmitting antennas when there is no channel state information available at the transmission side (e.g. broadcasting systems)
}

it is shown that the data path transmitted with QPSK and code rate $1 / 2$ can achieve reception at BB FER $1 \%$ with approximately $3.0 \mathrm{~dB}$ of CNR for $10 \mathrm{~Hz}$ of Doppler and with $2.5 \mathrm{~dB}$ for $80 \mathrm{~Hz}$. From Fig. 10 we can see that reception of the L1-post signalling at FER 1\% can be achieved with approximately $1 \mathrm{~dB}$ lower.

The previous results represent the worst case scenario in which the data path is transmitted with the most robust constellation (QPSK) and code rate (1/2). If the data path is transmitted with a less robust configuration, the difference between the robustness of the signalling and the data path increases. For example, if 16QAM and code rate 1/2 are used for the data path, according to Fig. 9, reception at BB FER $1 \%$ is possible with $8 \mathrm{~dB}$ of CNR for $10 \mathrm{~Hz}$ of Doppler and with $7.5 \mathrm{~dB}$ for $80 \mathrm{~Hz}$. In this case, it is possible to transmit the L1-post signalling with QPSK instead of BPSK and still achieve a higher robustness than the data path.

The main difference between the results presented here and those from [10], is the utilization of Genie aided demapping in the later case. Genie aided demapping emulates the ultimate performance of iterative decoding and demapping. In conjunction with rotated constellations, Genie aided demapping can achieve gains up to several $\mathrm{dBs}$ depending on the constellation and code rate [11]. On the other hand, rotated constellations are not allowed for the L1 signalling and hence, the utilization of Genie aided does not provide any significant gain. Because of this, the results presented in [10] depict a pessimistic performance of the signalling path compared to the data path. Due to the fact that Genie aided demapping does not represent the true performance expected with actual hardware, the results shown in this paper are presumably closer to real life performance.

\section{Shadowing - Data Path}

Fig. 11 illustrates the performance of the system when shadowing is also taken into account. We have represented the results for increasing values of inter-frame interleaving that correspond to interleaving durations up to approximately 10 seconds. In each configuration we have employed the 


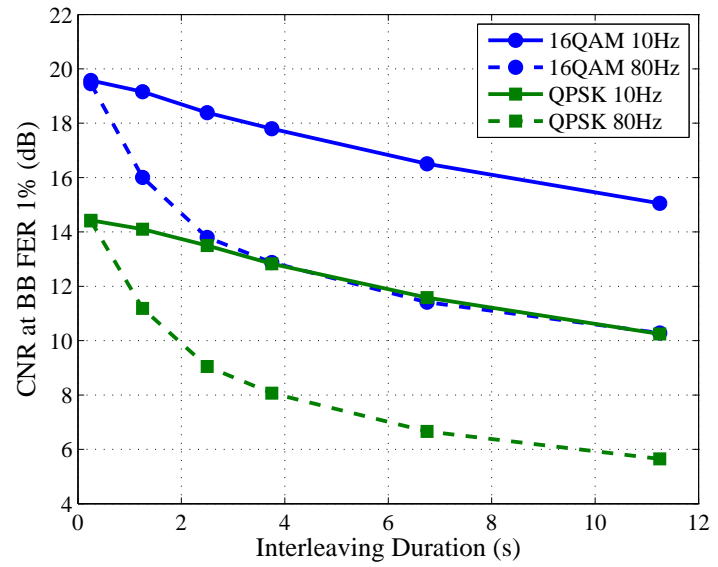

Fig. 11. CNR at BB FER 1\% for different configurations of inter-frame interleaving and maximum sub-slicing. Reception escenario with $d_{c o r r}=20 \mathrm{~m}$ and $\sigma=5.5 \mathrm{~dB}$.

maximum sub-slicing value allowed by the standard. Contrary to the fast fading scenario, the utilization of inter-frame interleaving achieves important gains in the presence of shadowing. In particular, $10 \mathrm{~s}$ of interleaving provide a gain of $4 \mathrm{~dB}$ in the case of $10 \mathrm{~Hz}$ of Doppler and a gain of $9 \mathrm{~dB}$ in the case of $80 \mathrm{~Hz}$. From Fig. 11 we can see that the Doppler frequency has a significant impact in the performance of interframe interleaving. Users moving at higher velocities employ less time passing through the shadow of building and trees and experiment a higher time diversity as a result. It must be noted that although the use of inter-frame interleaving involves a significant improvement in shadowing scenarios, the lack of fast zapping support at the physical layer of DVB-T2 limits its utilization due to channel change time issues.

\section{Shadowing - Signalling Path}

The frame error curves of the L1-post signalling in the presence of fast fading and shadowing are plotted in Fig. 12. According to the results, the gain due to L1 repetition is approximately $3 \mathrm{~dB}$ for $10 \mathrm{~Hz}$ of Doppler and $4 \mathrm{~dB}$ for $80 \mathrm{~Hz}$. Although this represents a very significant gain, if we compare these results with Fig. 11, we can see that the use of L1 repetition no longer ensures a higher robustness of the signalling over the data path. The utilization of interframe interleaving in high mobility scenarios (i.e. vehicular reception) may result in very large gains that cannot be achieved by means of L1 repetition. It must be noted that low diversity scenarios do not present this problem, as the gain achieved by means of inter-frame interleaving does not usually provide a higher robustness than the signalling path.

For example, in the case of $80 \mathrm{~Hz}$ of Doppler, reception of L1-post signalling at FER 1\% can be achieved with $9 \mathrm{~dB}$ of CNR if BPSK and L1 repetition is used. In the case of QPSK and code rate $1 / 2$, interleaving durations longer than $2 \mathrm{~s}$ in the data path allow the reception at BB FER $1 \%$ with CNR values lower than $9 \mathrm{~dB}$. On the contrary, if a less robust transmission mode such as 16QAM and code rate 1/2 is employed for the data path, the use of inter-fame interleaving cannot achieve reception with a CNR lower than $9 \mathrm{~dB}$.

In scenarios where the robustness of the signalling path is not sufficient compared to the data path, it is necessary to rely on in-band signalling in order to ensure the continuous reception of the service. This way, the L1 signalling can benefit from inter-frame interleaving and possess the same robustness as the data. Nevertheless, reception of the L1 signalling in the P2 symbols is still necessary the first time the user starts receving the service or after a signal outage. In this case, the user has to wait until being in good reception conditions before reception can begin or resume after the event of a signal outage. From Fig. 12, we can see that reception of the L1-post signalling with L1 repetition is still possible at FERs lower than $10 \%$ with a CNR higher than $5 \mathrm{~dB}$.

\section{E. Shadowing - Upper Layer FEC}

In Fig. 13 we show the performance of DVB-T2 when ULFEC is used to provide long time interleaving. In particular, we have consider the protection of IP packets by means of MPE-iFEC. We have used this as an example of how ULFEC protection could be implemented in DVB-T2 in order to validate the performance of the system. For the simulations we have used a configuration of 16QAM, code rate $1 / 2$ and maximum sub-slicing.

From the figures we can see that in this case the gain depends not only on the interleaving duration but also on the code rate used for UL-FEC. According to the results, similar gains than those achieved at the physical layer can be obtained with an UL-FEC code rate of $1 / 2$. It must be noted that the use of code rate 1/2 for UL-FEC with 16QAM and code rate 1/2 at the physical layer results in an spectral efficiency of 1 bit per cell (bpc). This is similar to the spectral efficiency obtained with QPSK and code rate 1/2 at the physical layer without ULFEC protection. If we compare the results presented in Fig. 13 with those of Fig. 11, in the case of $80 \mathrm{~Hz}$ of Doppler, the utilization of UL-FEC enables the reception at lower CNR values with the same spectral efficiency. For example, with 10 seconds of interleaving, a configuration of 16QAM and code rate $1 / 2$ at the physical layer with UL-FEC code rate $1 / 2$ can achieve reception at IP PER $1 \%$ with $10.5 \mathrm{~dB}$ of CNR. A configuration of QPSK and code rate 1/2 at the physical layer without UL-FEC requires approximately $14.5 \mathrm{~dB}$ of CNR.

On the contrary, UL-FEC may not provide any gain for the same spectral efficiency in low diversity scenarios. If we perform the same comparison as before, we can see that in the case of $10 \mathrm{~Hz}$ of Doppler, the configuration with UL-FEC requires approximately $15 \mathrm{~dB}$ of $\mathrm{CNR}$, which is $0.5 \mathrm{~dB}$ higher than the case with no UL-FEC.

\section{CONCLUSIONS}

In this paper we have investigated the use of time diversity in mobile DVB-T2 systems. The standard incorporates a very flexible time interleaving scheme that allows multiple tradeoffs in terms of time diversity, latency and power saving by means of inter-frame interleaving, frame hopping and sub-slicing. Inter-frame interleaving can provide interleaving durations up to several seconds at the expense of a significant increase in 

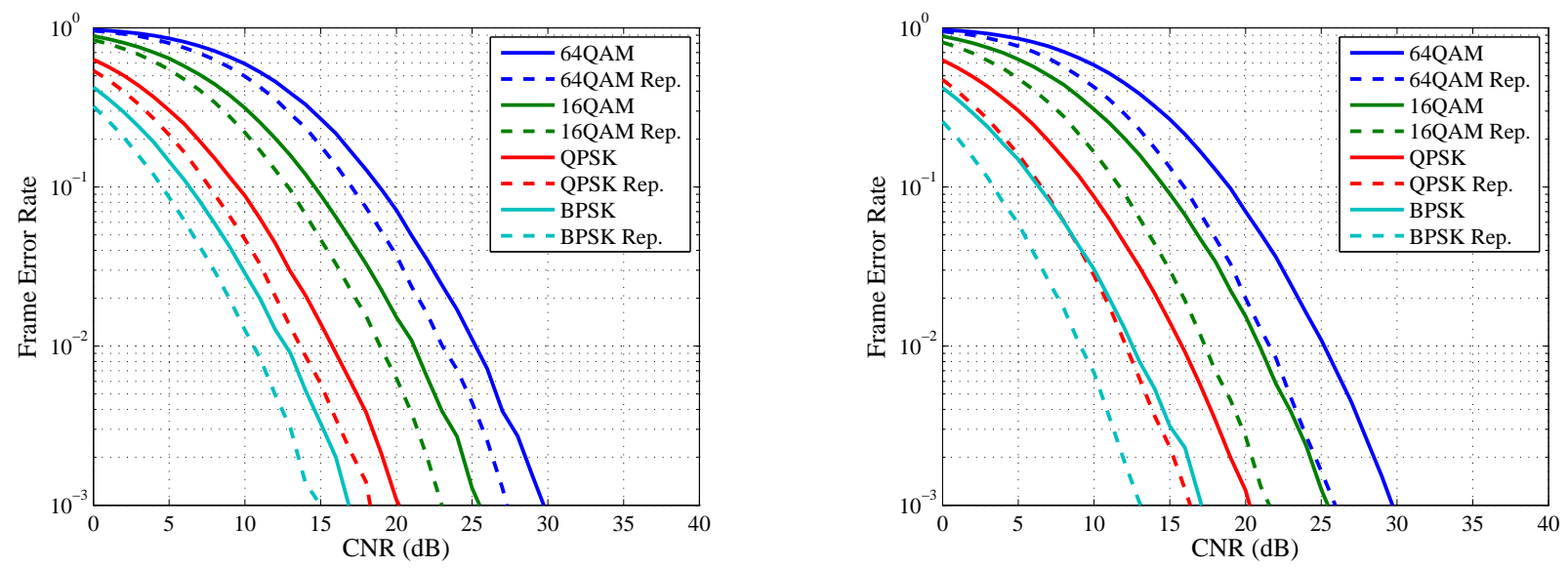

Fig. 12. T2 frame error rate curves of the L1-post signalling in a reception escenario with $d_{\text {corr }}=20 \mathrm{~m}, \sigma=5.5 \mathrm{~dB}, f_{d}=10 \mathrm{~Hz}$ (left) and $f_{d}=80 \mathrm{~Hz}$ (right).
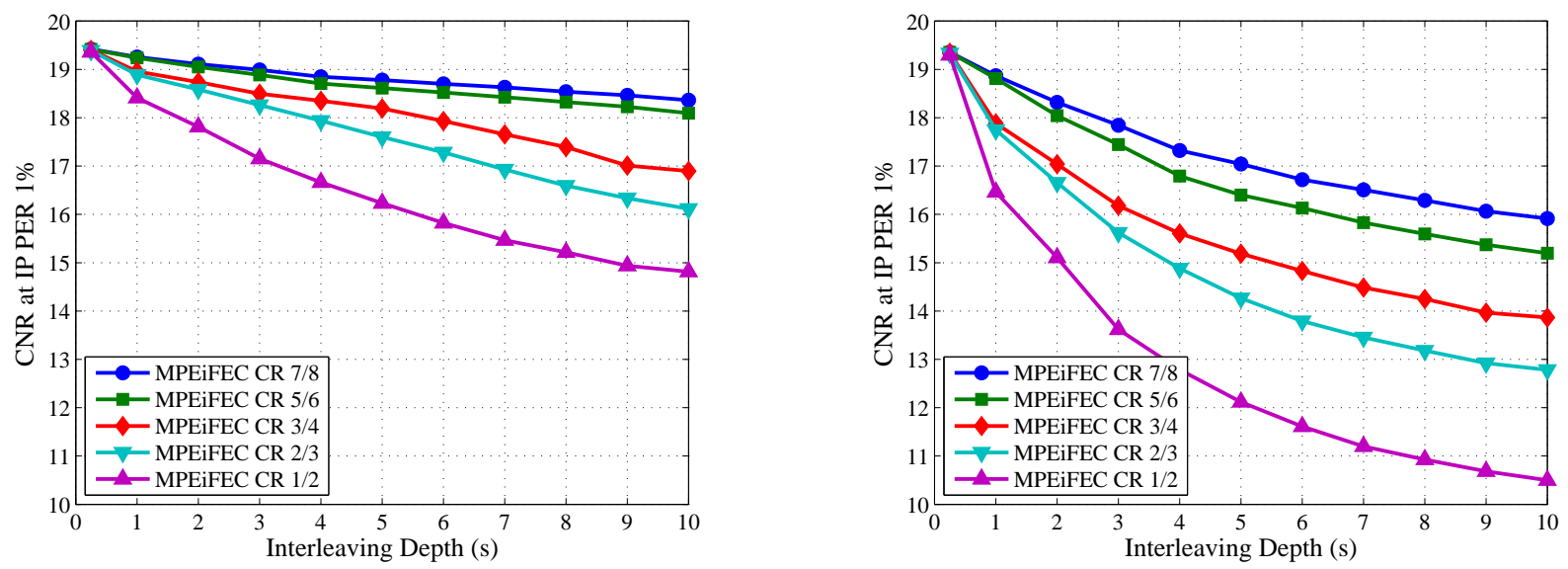

Fig. 13. CNR at IP FER 1\% for different configurations of MPEiFEC. DVB-T2 physical layer with 16QAM, code rate 1/2 and maximum sub-slicing. Reception escenario with $d_{\text {corr }}=20 \mathrm{~m}, \sigma=5.5 \mathrm{~dB}, f_{d}=10 \mathrm{~Hz}$ (left) and $f_{d}=80 \mathrm{~Hz}$ (right).

the channel change time. On the other hand, the use of subslicing results in an important improvement of the intra-frame interleaving by transmitting the information in a more even way within the T2 frames. Although the utilization of subslicing does not impact the channel change time in a significant way, it may reduce the power saving capabilities of DVB-T2 receivers. Lastly, frame hopping can enable power saving even when the information is transmitted continuously inside the T2 frames by means of sub-slicing.

Our investigation shows that the utilization of sub-slicing is highly benefitial in fast fading scenarios, and can achieve gains up to $4 \mathrm{~dB}$ in the case of intra-frame interleaving. Inter-frame interleaving on the other hand can only obtain gains up to 1 $\mathrm{dB}$ and its utilization in this case seems better suited to low diversity scenarios. In order to evaluate the performance of the future T2-mobile especification, we have also considered reception with reduced time de-interleaving (TDI) memory. In particular, we have considered half of the TDI memory defined in the DVB-T2 standard (i.e. $2^{18}$ ), which is the amount mandated in T2-mobile. The results show that the reduction in the TDI memory does not degrade the system performance as long as sub-slicing is employed. Otherwise, a degradation up to $1 \mathrm{~dB}$ can be expected in low Doppler scenarios.

As opposed to the case of fast fading, the utilization of inter-frame interleaving achieves very important gains in shadowing scenarios. Specifically, gains up to $4 \mathrm{~dB}$ and $9 \mathrm{~dB}$ can be obtained with $10 \mathrm{~s}$ of interleaving depending on the user mobility. Nevertheless, the lack of fast zapping support at the physical layer prevents the utilization of inter-frame interleaving for long interleaving durations due to channel change time issues. In order to provide long time interleaving with fast zapping, we investigate the utilization of upper layer forward error correction (UL-FEC). The results show that the use of UL-FEC can provide important gains in high diversity scenarios.

Regarding the robustness of the signalling in mobile channels, our simulations show that the utilization of L1 repetition and BPSK (Binary Phase Shift Keying) modulation in the signalling path ensure a higher robustness than the data path in the case of fast fading. If a less robust configuration than QPSK and code rate $1 / 2$ is employed for the data path, it may be possible to use higher order constellations than BPSK for 
the signalling path and still achieve a higher robustness.

On the contrary, the robustness of the L1 signalling carried in the P2 symbols may not be sufficiently high in shadowing escenarios when compared to the data path. Due to the large gains achieved by inter-frame interleaving, the use of L1 repetition may no longer ensure a higher robustness when the data path is transmitted with the most robust transmission modes. In this case, the transmission of the L1 signalling has to rely on in-band signalling to ensure the continuous reception of the service. Since the reception of the regular L1 signalling transmitted in the P2 symbols is still necessary at the beginning of the reception or after a signal outage, the user does not have other choice but to wait until being in good channel conditions before it is possible to begin or resume the service.

\section{REFERENCES}

[1] Frame structure channel coding and modulation for a second generation digital terrestrial television broadcasting system (DVB-T2), ETSI Std. EN 302755 v1.2.1, 2011.

[2] Implementation guidelines for a second generation digital terrestrial television broadcasting system (DVB-T2), ETSI Std. TR 102831 v0.10.4, 2010.

[3] L. Vangelista et al., "Key technologies for next-generation terrestrial digital television standard DVB-T2,' IEEE Communications Magazine, vol. 47, no. 10, p. 146-153, 2009.

[4] H. Fuchs and N. Färber, "Optimizing channel change time in IPTV applications," Proc. IEEE Broadband Multimedia Systems and Broadcasting, Las Vegas, USA, Mar. 2008.

[5] D. Gozálvez, D. Gómez-Barquero and N. Cardona, "Performance evaluation of the MPE-iFEC sliding RS encoding for DVB-H streaming services," Proc. IEEE Personal, Indoor and Mobile Radio Conference, Cannes, France, Sep. 2008.

[6] D. Gómez-Barquero, D. Gozálvez and N. Cardona, "Application layer FEC for mobile TV delivery in IP datacast over DVB-H systems," IEEE Transactions on Broadcasting, vol. 55, no. 2, p. 396-406, 2009.

[7] Upper Layer Forward Error Correction for DVB Systems (ULFEC), ETSI Std. TR 102993 v1.1.1, 2011.

[8] Multi-Protocol Encapsulation - inter-burst Forward Error Correction (MPE-iFEC), ETSI Std. TS 102772 v1.1.1, 2010.

[9] D. Gozálvez, D. Vargas, D. Gómez-Barquero and N. Cardona, "Performance evaluation of DVB-T2 time interleaving in mobile environments," Proc. IEEE Vehicular Technology Conference, Ottawa, Canada, Sep. 2010.

[10] T. Jokela, M. Tupala and J. Paavola, "Analysis of physical layer signaling transmission in DVB-T2 systems," IEEE Transactions on Broadcasting, vol. 56, no. 3, p. 410-417, 2010.

[11] C. Abdel Nour and C. Douillard, "Rotated QAM constellations to improve BICM performance for DVB-T2," Proc. IEEE International Symposium on Spread Spectrum Techniques and Applications, Bologna, Italy, Aug. 2008.

[12] S. M. Alamouti, "A simple transmit diversity technique for wireless communications," IEEE Journal on Selected Areas in Communications, vol. 16, no. 8, pp. 1451-1458, 1998.

[13] Implementation guidelines for DVB handheld services, ETSI Std. TR 102377 v1.4.1, 2009.

[14] M. L. Alberi Morel et al., "Performance evaluation of channel change for DVB-SH streaming services," Proc. IEEE International Conference on Communications, South Africa, May 2010.

[15] Guidelines for the Implementation for Satellite Services to Handheld devices (SH) below 3GHz, ETSI Std. TS 102584 v1.2.1, 2011.

[16] EUREKA/CELTIC WingTV Project web site [http://projects.celtic initiative.org/WING-TV/].

[17] P. Moss, "DVB-NGH channel models," TM-NGH063, 2010. 